OPEN ACCESS

Edited by:

Joyce S. Pang,

Nanyang Technological University,

Singapore

Reviewed by:

Manyu Li,

University of Louisiana at Lafayette,

United States

Nestor D. Kapusta,

Medical University of Vienna, Austria

${ }^{*}$ Correspondence:

Lian Jin

13501913934@126.com

Ying Wang

yingwangxun@163.com

Yong $\mathrm{Cai}$

caiyong202028@hotmail.com

tThese authors have contributed equally to this work and share first authorship

Specialty section:

This article was submitted to Gender, Sex and Sexualities, a section of the journal

Frontiers in Psychology

Received: 01 October 2021

Accepted: 17 January 2022

Published: 10 February 2022

Citation: Liu Y, Wang R, Chang R, Wang $H$, $X u L, X u C, Y u X$, Liu S, Chen H, Chen $Y$, Jin L, Wang $Y$ and Cai $Y$ (2022) Perceived

Burdensomeness, Thwarted Belongingness, and Social Exclusion

in Transgender Women:

Psychometric Properties of the Interpersonal Needs Questionnaire.

Front. Psychol. 13:787809.

doi: 10.3389/fpsyg.2022.787809

\section{Perceived Burdensomeness,} Thwarted Belongingness, and Social Exclusion in Transgender Women: Psychometric Properties of the Interpersonal Needs Questionnaire

\author{
Yujie Liu't, Rongxi Wang ${ }^{1 \dagger}$, Ruijie Chang ${ }^{1}$, Huwen Wang ${ }^{2}$, Lulu Xu ${ }^{1}$, Chen $X u^{1}$, Xiaoyue $Y u^{1}$, \\ Shangbin Liu', Hui Chen ${ }^{1}$, Yingjie Chen ${ }^{1}$, Lian Jin ${ }^{3 *}$ Ying Wang ${ }^{1 *}$ and Yong Cai ${ }^{1 *}$

\begin{abstract}
'School of Public Health, Shanghai Jiao Tong University School of Medicine, Shanghai, China, '2Jockey Club School of Public Health and Primary Care, The Chinese University of Hong Kong, Hong Kong SAR, Hong Kong, China, ${ }^{3}$ Ban Song Yuan Road Community Health Service Centre, Shanghai, China
\end{abstract}

Transgender women (TGW) experience serious psychiatric problems and high suicide rates. According to the interpersonal theory of suicide, thwarted belongingness and perceived burdensomeness play major roles in suicidality and can be measured by the Interpersonal Needs Questionnaire (INQ). However, no study has validated the use of the INQ in TGW. This study aimed to examine the psychometric properties of the INQ among TGW. We recruited 198 TGW (mean age 38.47 years) from Shenyang, China, using snowball sampling. The construct validity of the INQ was assessed through factor analysis, and convergent and divergent validity were examined through a structural equation model with other psychosocial factors. The construct validation analysis supported a three-factor model (perceived burdensomeness, thwarted belongingness, and social exclusion) with satisfactory fit indices: $\chi^{2} / \mathrm{df}=1.54, \mathrm{RMSEA}=0.052, \mathrm{CFI}=0.931, \mathrm{TLI}=0.916, \mathrm{SRMR}=0.053$. The thwarted belongingness was significantly associated with self-esteem and social support, and the social exclusion was significantly associated with loneliness, depression, entrapment, and defeat, suggesting satisfactory convergent and divergent validity for the three-factor model. The present findings indicate that for TGW, high social exclusion is important in assessing perceived interpersonal needs, while the notable deviation from previous two-factor model warrants further study.

Keywords: Interpersonal Needs Questionnaire, transgender women, psychometric property, suicide, social exclusion

\section{INTRODUCTION}

Transgender women (TGW) are those who were born with a male biological sex but have a female psychological sex and self-perception, regardless of medical interventions, such as gender reassignment surgery and hormone injections (Poteat et al., 2015; Becasen et al., 2019; Shi and Cai, 2020). A meta-analysis in 2015 showed that, considering only those seeking gender 
affirmation-related treatment at specialty clinics, transgender people represented about 4.6 per 100,000 of the total population globally, with 6.8 per 100,000 TGW and 2.6 per 100,000 transgender men. If all people questioning their gender were considered transgender, $0.5 \% \sim 1.3 \%$ of the global population are TGW and $0.4 \% \sim 1.2 \%$ are transgender men (Winter et al., 2016). The TGW population suffers from widespread discrimination, especially gender-related discrimination at 47.5\% (Becasen et al., 2019). Meanwhile, TGW experience serious mental health problems. A study reported that the depression rate of TGW was as high as $64 \%$ (Center for Epidemiologic Studies Depression Scale, CESD scale score $\geq 16$; Nemoto et al., 2014), four times higher than the general population (Hyde et al., 2013). Another study in China reported approximately half the sample experienced higher levels of loneliness $(51.1 \%)$ or was clinically depressed (49.1\%; Shi and Cai, 2020). Furthermore, suicide rates are also high among TGW, with a review showing that suicide attempts in this population were $24.8 \%$ (95\% CI: $18.0-33.2 \%$; Becasen et al., 2019). In a US study, $41 \%$ of TGW reported having attempted suicide, compared with a frequency of $1.6 \%$ in the general population (Winter et al., 2016).

Death by suicide causes great pain to those close to them. The mechanisms of suicide have been studied for many years, and several theories have been proposed to explain it. The Interpersonal Theory of Suicide suggested that suicide occurs by the simultaneous presence of suicidal ideation and acquired capacity of self-harm and suicidal ideation results from unmet interpersonal needs (Joiner, 2005; Van Orden et al., 2008). According to the theory, various indices of social isolation, such as loneliness and low social support, are associated with suicide because they indicate that the need to belong has been thwartedthwarted belongingness. Family dissension and unemployment are associated with suicide since they are probably to induce perception of burdensomeness on others-perceived burdensomeness. Unmet interpersonal needs may in turn intensify low self-esteem, loneliness, lack of social support, and depression (Van Orden et al., 2010, 2012; Park and Kim, 2019).

Thwarted belongingness and perceived burdensomeness are proximal and sufficient causes of suicidal thoughts and can be measured by the Interpersonal Needs Questionnaire (INQ) developed by Joiner and Kimberly (Joiner, 2005; Van Orden et al., 2008). INQ has been validated among college students (Becker et al., 2020; Loas et al., 2021), young male adults (Teo et al., 2018), community-dwelling older persons (Shim et al., 2021), current firefighters (Chu et al., 2016), clinically depressed and suicidal youth (Hunt et al., 2020) and military veterans (O'Connor et al., 2017), and translated to different languages (Podlogar et al., 2017; Silva et al., 2018; Park and Kim, 2019; Iliceto et al., 2021; Lai and Boag, 2021). While the two-factor structure-thwarted belongingness and perceived burdensomeness-showed reliable and acceptable model fit to college students, older people, and firefighters, studies among clinically depressed and suicidal youth (Hunt et al., 2020) and migrant workers (Wang et al., 2021a) validated a better model fit for three dimensions of the INQ. These particular groups like TGW and suicidal youth shared similar experiences of loneliness and as Quintin A. Hunt suggested, loneliness is not accurately measured as through the TB subscale (Hunt et al., 2020). Meanwhile, TGW reported more experiences of family rejection, discriminatory comments from family members, alienation from friends, unemployment, beatings, rape, etc. (Poteat et al., 2017). Given the traditional conservative culture in China, transgender people are excluded from the mainstream of society which indicated that the belonging need for them may be different from other population. It is reasonable to question that whether a two-factor structure of INQ remains unchanged in TGW. Furthermore, early identification of suicidal ideation before suicide attempt can help prevent suicide considering the high suicide rate among this population.

Although the Chinese version of the INQ has been widely used to measure interpersonal needs and predict suicidal ideation, its psychometric properties remained unclear in TGW population. The purpose of the present study is twofold: to explore the dimension structure of INQ through exploratory and confirmatory factor analysis (CFA) and to assess the convergent and divergent validity of INQ among TGW using structure equation model, determining whether the INQ is a reliable and valid assessment tool among TGW in China.

\section{MATERIALS AND METHODS}

\section{Participants}

The cross-sectional survey of TGW in Shenyang, China was conducted from April to July 2017. A snowball sampling method was used for recruitment with the help of a non-governmental organization dedicated to improving the physiological and psychological health of TGW. An initial set of five eligible participants completed the survey, then they were asked to recommend others to be recruited into the study. The participants forwarded the questionnaire survey in this way until they could not identify others who meet the inclusion criteria. The inclusion criteria were: (1) aged 18 years or older; (2) had male biological sex; (3) had female psychological sex; (4) had the ability to understand the questionnaire; and (5) informed about the study procedure and signed written informed consent.

All participants were informed about the assurance of anonymity during the survey, the right to withdraw at any time, and that there would be no consequence of refusing the survey. A total of 198 TGW were finally recruited in the study. Participants' ages ranged from 18 to 62 years, with a mean age of 33.47 years.

\section{Measures}

\section{Chinese Version of the INQ-15}

The 15-item INQ, adapted from the original 25-item INQ, was designed to measure suicide-related interpersonal needs (Van Orden et al., 2012). The two-factor measurement model of the INQ-15 has been validated in several previous research, which measures two dimensions: perceived burdensomeness and thwarted belongingness (Amini-Tehrani et al., 2021; Iliceto et al., 2021). In the INQ-15, each item is assessed on a 7-point Likert scale from "not true for me at all" to "very true for me." 
Total score of the INQ-15 is between 0 and 105, with higher scores indicate severer unmet interpersonal needs. The Chinese version of INQ-15, which was translated by Chen, demonstrated good internal consistency among college students, with Cronbach's alpha values ranging from 0.72 to 0.93 for perceived burdensomeness and 0.78 to 0.91 for thwarted belongingness (Li et al., 2015; Lai and Boag, 2021).

\section{Chinese Version of the RSES}

The Rosenberg Self-Esteem Scale (RSES) was developed by Rosenberg to measure the degree of self-esteem (Rosenberg, 1989). The scale consists of ten items that evaluate two dimensions of self-esteem: self-competence and self-liking. Total scores of the RSES range from 10 to 40, and higher scores indicate higher level of self-esteem. The Chinese version of the RSES was translated by Ji and exhibited adequate internal consistency in migrants and adolescents (Cronbach's alpha $=0.81$; Liang et al., 2020; Wang et al., 2021b).

\section{Chinese Version of the 8-Item UCLA Loneliness Scale (ULS-8)}

The 8-item ULS is a self-reported measure of perceived loneliness, which was refined from the original 20-item ULS by Hays (Hays and DiMatteo, 1987). In the ULS-8, respondents were asked about the frequency of possible negative experiences associated with social relationships (i.e., "I lack companionship"), ranging from 1 ("never") to 4 ("always"). Higher scores of ULS-8 indicate greater level of perceived loneliness. The Chinese version of the ULS-8 was translated by Zhou and demonstrated good internal consistency in adolescents and older adults (Cronbach's alpha $=0.831-0.878$; Zhou et al., 2012; Xu et al., 2018).

\section{Chinese Version of the 9-Item PHQ-9}

The Patient Health Questionnaire (PHQ-9) is an instrument for assessing the severity of depressive symptoms, which was derived from the depression module from the self-administered mental health diagnostic instrument PHQ (Kroenke et al., 2001). The scale consists of nine 4-point Likert-type items from 0 ("not at all") to 4 ("nearly every day"), with higher scores indicate severe depressive symptoms. The Chinese version of the PHQ-9 has shown good internal consistency among adolescents, with Cronbach's alpha value between 0.84 and 0.86 (Tsai et al., 2014; Leung et al., 2020).

\section{Chinese Version of the ES}

The 16-item Entrapment Scale (ES) was developed by Gilbert and Allan to measure the extent an individual feels imprisoned or trapped by unbearable thoughts, feelings or circumstances (Gilbert and Allan, 1998). The scale consists of two dimensions: external entrapment (EE) and inner entrapment (IE). In the $\mathrm{ES}$, each item is assessed on a 5-point Likert scale from 0 ("not at all like me") to 4 ("extremely like me"), and higher scores indicate greater feeling of entrapment. The Chinese version of the ES has exhibited good internal consistency among college students (Cronbach's alpha $=0.94$ for EE, 0.93 for IE; Gong et al., 2019).

\section{Chinese Version of the DS}

The 16-item ES was developed by Gilbert and Allan to measure the sense of failed struggle and low social rank (Gilbert and Allan, 1998). The scale consists of two dimensions: decadence and low sense of achievement. In the Defeat Scale (DS), each item is assessed on a 5-point Likert scale from 0 ("never") to 4 ("always"), and higher scores indicate greater feeling of entrapment. The Chinese version of the DS has exhibited good internal consistency among college students (Cronbach's alpha $=0.933$; Tang et al., 2019).

\section{Chinese Version of the MSPSS}

The multidimensional scale of perceived social support (MSPSS) is a 12 -item self-reported measure of perceived social support from three sources: family, friends, and significant others. Respondents were asked to report their agreement with items (i.e., "My family really tries to help me") on a 7-point Likert scale (Zimet et al., 1988). Total scores of the MSPSS are between 12 and 84, with higher scores indicate higher perceived social support. The Chinese version of the DS has demonstrated adequate internal consistency among college students (Cronbach's alpha $=0.86$ for family; 0.89 for friends; 0.86 for significant other; Zhang et al., 2018).

\section{Statistical Analysis}

All analyses were performed using Mplus version 7.4 and $\mathrm{R}$ 4.0.0. Descriptive statistics were conducted for INQ items and psychosocial factors using R 4.0.0. Reliability of the INQ was assessed by internal consistency using Cronbach's alpha coefficient, with a coefficient of greater than 0.7 indicates good internal consistency (Nunnally and Bernstein, 1994).

Exploratory factor analysis and CFA were conducted to examine the factor structure of the scale using Mplus version 7.4. Maximum likelihood estimation with robust standard errors with geomin oblique rotation was utilized because the majority of INQ items were non-normally distributed. Additionally, the INQ was analyzed via Item Response Theory (IRT) models to obtain more complex information about the psychometric properties of the individual assessment items. A series of fit indices were used to examine the goodness of fit of the model: chi-square $\left(\chi^{2}\right)$, standardized root mean square residual (SRMR), comparative fit index (CFI), Tucker-Lewis index (TLI), and the root mean squared error of approximation (RMSEA). The assessment criteria for each index were: $\chi^{2} / \mathrm{df}<3 \quad(p>0.05)$, SRMR $<0.08$, CFI $>0.9$, TLI $>0.9$, RMSEA $<0.08$ (McDonald and Ho, 2002).

The statistical power $(\pi)$ of the CFA model was estimated by Monte Carlo simulation. In Monte Carlo simulation, a hypothesized population value for each model parameter was set identical to the established CFA model. The model was estimated based on a large number of randomly generated samples. Muthén and Muthén suggested that the following 
criteria should be meet to achieve a desirable level of $\pi(0.80)$ with a certain sample size: (1) parameter and standard error bias $<10 \%$; (2) standard error bias for the parameter of interest $<5 \%$; and (3) coverage (the proportion of samples for which the $95 \%$ confidence interval contains the parameter value) between 0.91 and 0.98 (Muthén and Muthén, 2002).

A structural equation model with the six psychosocial variables and INQ was constructed using Mplus version 7.4. All the observed variables were regressed onto the latent factors of the INQ-15 in the model. The magnitude of the regression coefficients indicated whether dissimilar constructs of the scale could be distinguished by different observed variables.

\section{Ethics}

The study procedures were carried out according to the Declaration of Helsinki. The Institutional Review Board of the School of Public Health, Shanghai Jiao Tong University School of Medicine approved this study. All participants were informed about the study, and all provided informed consent.

\section{RESULTS}

\section{Description of the INQ-15}

Descriptive statistics (i.e., number, mean, standard error, standard deviation, variance, skewness, kurtosis, and range) and intercorrelations between the 15 items for the whole sample are presented in Table 1.

\section{Construct Validity}

\section{The Exploratory Factor Analysis}

The results of EFA with 1-4 factors yielded three eigenvalues greater than one (Table 2). Both the three-factor model and the four-factor model displayed good model fits on $x^{2} /$ df, RMSEA, CFI, TLI, and SRMR. After examining factor structures of the two models, the three-factor solution had better indicators for each factor and was finally decided (Table 3). The pattern of the loadings for the model indicated that the first six items $(1 / 2 / 3 / 4 / 5 / 6)$ loaded onto the "perceived burdensomeness" factor and item 7/8/10/13/14/15 loaded onto the "thwarted belongingness" factor. The remaining three items (9/11/12) loaded onto a third factor which was named "social exclusion."

\section{The Confirmatory Factor Analysis}

Estimated parameters for the three-factor model (standardized estimated factor loadings, standard error, covariances, and $R^{2}$ values) are presented in Table 4. All items significantly loaded onto the anticipated latent factors revealed by the EFA, with $R^{2}$ ranging from 0.195 to 0.640 . The three factors (perceived burdensomeness, thwarted belongingness, and social exclusion) were distinct but closely correlated, with perceived burdensomeness and social exclusion positively correlated with each other $(r=0.641)$ while negatively correlated with thwarted belongingness $(r=-0.338 ; r=-0.474)$.

\section{IRT Modeling and M2 Test}

The results of M2 test indicated good model fit of the IRT modeling: $\mathrm{RMSEA}=0.037(90 \% \mathrm{CI}=0.009-0.065), \mathrm{M} 2=11.94$ $(p=0.036)$.

\section{Statistical Power Estimation}

In Monte Carlo simulation, the parameter and standard error bias were $0.74 \%$ and $-2.58 \%$ for factor 1 and factor $2,-0.18 \%$ and $-1.85 \%$ for factor 1 and factor 3 , and $-0.21 \%$ and $-4.22 \%$ for factor 2 and factor 3 . All the parameter and standard error bias did not exceed 5\%, and all the coverage remained above the minimum threshold of 0.91 . Therefore, a sample size of 198 in this study was enough for a $\pi$ of 0.80 . Results of Monte Carlo simulation are presented in Supplementary Material.

\section{Reliability}

Cronbach's alpha values for the Chinese version of the INQ-15 were 0.848 for the total scale, 0.849 for perceived burdensomeness, 0.808 for thwarted belongingness, and 0.660 for social exclusion. The internal consistency was satisfactory for the total scale as well as the first two factors. Although social exclusion had a comparatively low Cronbach's alpha value, it is acceptable for a subscale with three items (Rust and Golombok, 1999).

\section{Convergent and Divergent Validity Description of Psychosocial Factors}

Descriptive statistics (i.e., number, mean, standard error, standard deviation, variance, skewness, kurtosis, and range) of the psychosocial factors (i.e., perceived social support, loneliness, depression, self-esteem, entrapment, and defeat) for the whole sample are presented in Table 5. Figure 1 showed the intercorrelations among different psychosocial factors, and interpersonal needs is positively correlated with loneliness $(r=0.49)$, depression $(r=0.56)$, entrapment $(r=0.62)$, defeat $(r=0.60)$, and negatively correlated with perceived social support $(r=-0.57)$.

\section{Convergent and Divergent Validity}

Figure 2 presented the results of the simultaneous regression of the six psychological factors on the three latent factors of perceived burdensomeness, thwarted belongingness, and social exclusion. The thwarted belongingness was associated with self-esteem and social support $(p<0.05)$; the social exclusion was associated with loneliness, depression, entrapment, and defeat $(\mathrm{p}<0.05)$; the perceived burdensomeness was associated with none of the six psychological factors.

\section{DISCUSSION}

The aim of the present study was to examine the structure and validate the use of the Chinese version of the INQ-15 among a sample of TGW. Although the INQ has been widely translated and used in different countries to measure interpersonal 
TABLE 1 | Descriptive statistics and inter-correlations of the interpersonal needs questionnaire items.

\begin{tabular}{|c|c|c|c|c|c|c|c|c|c|c|c|c|c|c|c|}
\hline & Item1 & Item2 & Item3 & Item4 & Item5 & Item6 & Item7 & Item8 & Item9 & Item10 & Item11 & Item12 & Item13 & Item14 & Item15 \\
\hline Item1 & 1.000 & $0.710 * *$ & $0.468^{* *}$ & $0.500 * *$ & $0.441 * *$ & $0.399 * *$ & $0.152 *$ & $0.207 * *$ & $0.229 * *$ & $0.165 *$ & $0.286 * *$ & $0.360 * *$ & $0.145 *$ & $-0.182^{*}$ & $0.228 * *$ \\
\hline Item2 & & 1.000 & $0.432 * *$ & $0.591 * *$ & $0.470 * *$ & $0.455 * *$ & $0.178 *$ & $0.287 * *$ & $0.213^{* *}$ & $0.184 * *$ & $0.400 * *$ & $0.395^{* *}$ & $0.203 * *$ & $0.251 * *$ & $0.199 * *$ \\
\hline Item3 & & & 1.000 & $0.577^{* *}$ & $0.511 * *$ & $0.452 * *$ & $0.236 * *$ & $0.280 * *$ & $0.297 * *$ & $0.224 * *$ & $0.409 * *$ & $0.376 * *$ & $0.179 *$ & $0.251 * *$ & $0.204 * *$ \\
\hline Item4 & & & & 1.000 & $0.532 * *$ & $0.550 * *$ & $0.181 *$ & $0.302 * *$ & $0.254 * *$ & $0.160 *$ & $0.369 * *$ & $0.479 * *$ & $0.182^{*}$ & $0.238^{* * *}$ & $0.250 * *$ \\
\hline Item5 & & & & & 1.000 & $0.553^{* * *}$ & $0.208^{* *}$ & $0.203^{* *}$ & $0.305 * *$ & $0.211^{* *}$ & $0.432^{* * *}$ & $0.436 * *$ & $0.206 * *$ & $0.225^{* * *}$ & $0.276 * *$ \\
\hline Item6 & & & & & & 1.000 & $0.256 * *$ & $0.211 * *$ & $0.309 * *$ & $0.182 *$ & $0.387 * *$ & $0.380 * *$ & $0.202 * *$ & $0.188 * *$ & $0.206 * *$ \\
\hline Item7 & & & & & & & 1.000 & $0.488^{* *}$ & $0.265^{* *}$ & $0.574 * *$ & $0.219 * *$ & $0.336^{* *}$ & $0.458^{* *}$ & $0.445^{* *}$ & $0.315^{* *}$ \\
\hline Item8 & & & & & & & & 1.000 & 0.104 & $0.388^{* *}$ & $0.296 * *$ & $0.279 * *$ & $0.399 * *$ & $0.423 * *$ & $0.391 * *$ \\
\hline Item9 & & & & & & & & & 1.000 & $0.299 * *$ & $0.441 * *$ & $0.398^{* *}$ & $0.314 * *$ & $0.291 * *$ & $0.219 * *$ \\
\hline Item10 & & & & & & & & & & 1.000 & $0.273^{* *}$ & $0.328^{* *}$ & $0.580 * *$ & $0.539 * *$ & $0.348 * *$ \\
\hline Item11 & & & & & & & & & & & 1.000 & $0.553^{* *}$ & $0.268^{* *}$ & $0.266^{* *}$ & $0.245 * *$ \\
\hline Item12 & & & & & & & & & & & & 1.000 & $0.283^{* *}$ & $0.324 * *$ & $0.332 * *$ \\
\hline Item13 & & & & & & & & & & & & & 1.000 & $0.543^{* * *}$ & $0.427^{* *}$ \\
\hline Item14 & & & & & & & & & & & & & & 1.000 & $0.550 * *$ \\
\hline Item15 & & & & & & & & & & & & & & & 1.000 \\
\hline$N$ & 198 & 198 & 198 & 198 & 198 & 198 & 198 & 198 & 198 & 198 & 198 & 198 & 198 & 198 & 198 \\
\hline Mean & 2.26 & 2.29 & 1.79 & 1.95 & 1.88 & 1.84 & 1.84 & 4.63 & 2.70 & 5.51 & 2.59 & 2.57 & 5.25 & 4.90 & 4.52 \\
\hline SE & 0.111 & 0.119 & 0.101 & 0.115 & 0.096 & 0.104 & 0.104 & 0.146 & 0.136 & 0.127 & 0.134 & 0.136 & 0.126 & 0.128 & 0.140 \\
\hline SD & 1.561 & 1.672 & 1.427 & 1.619 & 1.350 & 1.467 & 1.467 & 2.048 & 1.914 & 1.790 & 1.880 & 1.921 & 1.776 & 1.804 & 1.971 \\
\hline Var & 2.436 & 2.797 & 2.036 & 2.622 & 1.823 & 2.153 & 2.153 & 4.195 & 3.664 & 3.206 & 3.533 & 3.689 & 3.154 & 3.255 & 3.885 \\
\hline Skew & 1.029 & 1.191 & 2.074 & 1.770 & 1.636 & 1.979 & 1.979 & -0.384 & 0.822 & -1.094 & 0.926 & 0.965 & -0.855 & -0.521 & -0.308 \\
\hline Kurt & 0.220 & 0.554 & 3.956 & 2.276 & 2.175 & 3.391 & 3.391 & -1.048 & -0.563 & 0.183 & -0.284 & -0.297 & -0.114 & -0.601 & -0.978 \\
\hline Range & 6 & 6 & 6 & 6 & 6 & 6 & 6 & 6 & 6 & 6 & 6 & 6 & 6 & 6 & 6 \\
\hline
\end{tabular}

$S D$, Standard deviation; SE, Standard error; Var, Variance; Skew, Skewness; Kurt, Kurtosis.

$* p<0.05, * * p<0.01$. 
TABLE 2 | Model fit indices for the Interpersonal Needs Questionnaire.

\begin{tabular}{|c|c|c|c|c|c|c|c|c|c|}
\hline Factor & $\chi^{2}$ & df & Value of $p$ & RMSEA & $90 \% \mathrm{Cl}$ & CFI & TLI & SRMR & Eigenvalue \\
\hline 1 & 354.801 & 90 & $<0.005$ & 0.122 & $0.109-0.135$ & 0.612 & 0.547 & 0.119 & 4.964 \\
\hline 2 & 174.735 & 89 & $<0.005$ & 0.07 & $0.054-0.085$ & 0.874 & 0.852 & 0.069 & 2.324 \\
\hline 3 & 134.388 & 87 & 0.0008 & 0.052 & $0.034-0.069$ & 0.931 & 0.916 & 0.053 & 1.169 \\
\hline 4 & 134.124 & 85 & 0.0005 & 0.054 & $0.036-0.071$ & 0.928 & 0.911 & 0.053 & 0.917 \\
\hline
\end{tabular}

$\chi^{2}$. Chi-square; SRMR, Standardized root mean square residual; CFI, Comparative fit index; TLI, Tucker-Lewis index; RMSEA, Root mean squared error of approximation.

TABLE 3 | Factor structure of the Interpersonal Needs Questionnaire.

\begin{tabular}{|c|c|c|c|}
\hline & \multicolumn{3}{|c|}{ Three-factor model } \\
\hline & PB & TB & SE \\
\hline 1.Better off & 0.765 & -0.159 & 0.145 \\
\hline 2.Happier out me & 0.804 & -0.213 & 0.119 \\
\hline 3.Burden to society & 0.642 & -0.267 & 0.369 \\
\hline 4.Death as relief & 0.847 & -0.22 & 0.192 \\
\hline 5.Rid of me & 0.722 & -0.231 & 0.418 \\
\hline 6.Makes worse & 0.687 & -0.234 & 0.338 \\
\hline 7.Others care & -0.162 & 0.701 & -0.197 \\
\hline 8.1 belong & -0.324 & 0.673 & 0.002 \\
\hline 9.Rarely interact & 0.173 & -0.209 & 0.825 \\
\hline 10.Friends & -0.11 & 0.742 & -0.22 \\
\hline 11.Disconnected & 0.446 & -0.26 & 0.737 \\
\hline 12.Outsider & 0.496 & -0.385 & 0.578 \\
\hline 13.Turn to & -0.126 & 0.731 & -0.248 \\
\hline 14.Close to others & -0.209 & 0.783 & -0.222 \\
\hline 15.Daily interact & -0.289 & 0.666 & -0.179 \\
\hline
\end{tabular}

PB, Perceived burdensomeness; TB, Thwarted belongingness; SE, Social exclusion; S.E., Standard error. Bold: significant at $5 \%$ level.

TABLE 4 | Estimated parameters for the three-factor model.

\begin{tabular}{lcccc}
\hline & Estimated & S.E. & $\boldsymbol{p}$ & $\boldsymbol{R}^{\mathbf{2}}$ \\
\hline Perceived burdensomeness & & & \\
1.Better off & 0.686 & 0.065 & $<0.001$ & 0.471 \\
2.Happier out me & 0.730 & 0.060 & $<0.001$ & 0.533 \\
3.Burden to society & 0.614 & 0.084 & $<0.001$ & 0.377 \\
4.Death as relief & 0.800 & 0.039 & $<0.001$ & 0.640 \\
5.Rid of me & 0.694 & 0.061 & $<0.001$ & 0.482 \\
6.Makes worse & 0.657 & 0.074 & $<0.001$ & 0.432 \\
Thwarted belongingness & & & \\
7.Others care & 0.605 & 0.072 & $<0.001$ & 0.366 \\
8.I belong & 0.578 & 0.063 & $<0.001$ & 0.334 \\
10.Friends & 0.662 & 0.072 & $<0.001$ & 0.438 \\
13.Turn to & 0.672 & 0.069 & $<0.001$ & 0.452 \\
14.Close to others & 0.748 & 0.052 & $<0.001$ & 0.560 \\
15.Daily interact & 0.615 & 0.059 & $<0.001$ & 0.378 \\
Social exclusion & & & & \\
9.Rarely interact & 0.442 & 0.082 & $<0.001$ & 0.195 \\
11.Disconnected & 0.714 & 0.063 & $<0.001$ & 0.510 \\
12.Outsider & 0.742 & 0.069 & $<0.001$ & 0.551 \\
Covariances & & & & \\
SE WITH PB & 0.641 & 0.075 & $<0.001$ & \\
SE WITH TB & -0.474 & 0.093 & $<0.001$ & \\
PB WITH TB & -0.338 & 0.083 & $<0.001$ & \\
\hline
\end{tabular}

PB, Perceived burdensomeness; TB, Thwarted belongingness; SE, Social exclusion; S.E., Standard error. needs and predict suicidal ideation, few studies have examined the psychometric properties of INQ in Chinese people and none of them focused on TGW despite their high suicide rates (Lai and Boag, 2021; Wang et al., 2021a). Considering the special social and cultural status in which TGW live (Winter et al., 2016), it is necessary to determine whether the INQ is a reliable and valid instrument for assessing interpersonal needs in this population.

Our results from the exploratory factor analysis supported the validity of a three-factor model. This model had six indicators for the perceived burdensomeness dimension, six indicators for the thwarted belongingness dimension, and three indicators for the social exclusion dimension. This was notably deviated from most previous findings that the INQ had only two dimensions of perceived burdensomeness and thwarted belongingness (Amini-Tehrani et al., 2021; Iliceto et al., 2021). However, a study investigating the construct validity of the INQ among clinically depressed and suicidal youth extracted a third factor from the TB factor and named it "perceived isolation" (Hunt et al., 2020). This three-factor solution including an additional factor "social isolation" for the INQ was confirmed later in another sample of Chinese migrant workers (Wang et al., 2021a). One commonality of these two validation studies is that they focus on a specific population who tend to have poor interpersonal bonds as a result of environmental or emotional maladjustment. Similarly, for the TGW who suffer from widespread discrimination and exclusion owing to their gender incongruence (White Hughto et al., 2015), interpersonal alienation, and social disconnection exist as severe psychosocial problems. Therefore, it is reasonable to recognize social exclusion, which represents the extent an individual feels alienated from society, as a third dimension in the measurement of interpersonal needs among TGW. This was confirmed by the IRT modeling and M2 test.

We further examine the psychometric properties of the Chinese version of the INQ after identifying the three-factor model. In the simultaneous regressions of the six observed variables on the three latent variables, thwarted belongingness was correlated with self-esteem and social support, and social exclusion was correlated with loneliness, depression, entrapment, and defeat, while perceived burdensomeness was correlated with none of these variables. These results supported the divergent validity of the INQ, which extended previous findings that the INQ had adequate convergent validity but not divergent validity in an important way (Van Orden et al., 2012; Silva et al., 2018). Van Orden suggested that the low divergent validity may be explained by the fact that belongingness is closely related to a wide range of psychological 
TABLE 5 | Descriptive statistics of the psychosocial factors.

\begin{tabular}{|c|c|c|c|c|c|c|c|}
\hline & Mean & Median & SD & SE & Range & Skew & Kurtosis \\
\hline INQ & 37.85 & 36.00 & 14.797 & 1.052 & 63 & 0.442 & -0.262 \\
\hline INQ_PB & 12.02 & 10.00 & 6.887 & 0.489 & 36 & 1.472 & 2.384 \\
\hline INQ_TB & 17.98 & 17.00 & 7.996 & 0.568 & 35 & 0.426 & -0.206 \\
\hline INQ_SE & 7.85 & 7.50 & 4.410 & 0.313 & 18 & 0.573 & -0.571 \\
\hline Self_esteem & 26.33 & 26.00 & 2.746 & 0.195 & 22 & 0.048 & 2.379 \\
\hline Loneliness & 18.36 & 18.00 & 4.282 & 0.304 & 22 & 0.658 & -0.030 \\
\hline Depression & 6.55 & 5.00 & 5.699 & 0.405 & 27 & 1.250 & 1.856 \\
\hline Entrapment & 15.44 & 10.00 & 15.578 & 1.107 & 63 & 0.952 & -0.122 \\
\hline Defeat & 17.53 & 14.00 & 12.138 & 0.863 & 53 & 1.064 & 0.454 \\
\hline Social_support & 62.53 & 64.00 & 14.216 & 1.010 & 69 & -0.716 & 0.199 \\
\hline
\end{tabular}

INQ, Interpersonal need; PB, Perceived burdensomeness; TB, Thwarted belongingness; SE, Social exclusion; SD, Standard deviation; SE, Standard error; Skew, Skewness; Kurt, Kurtosis.

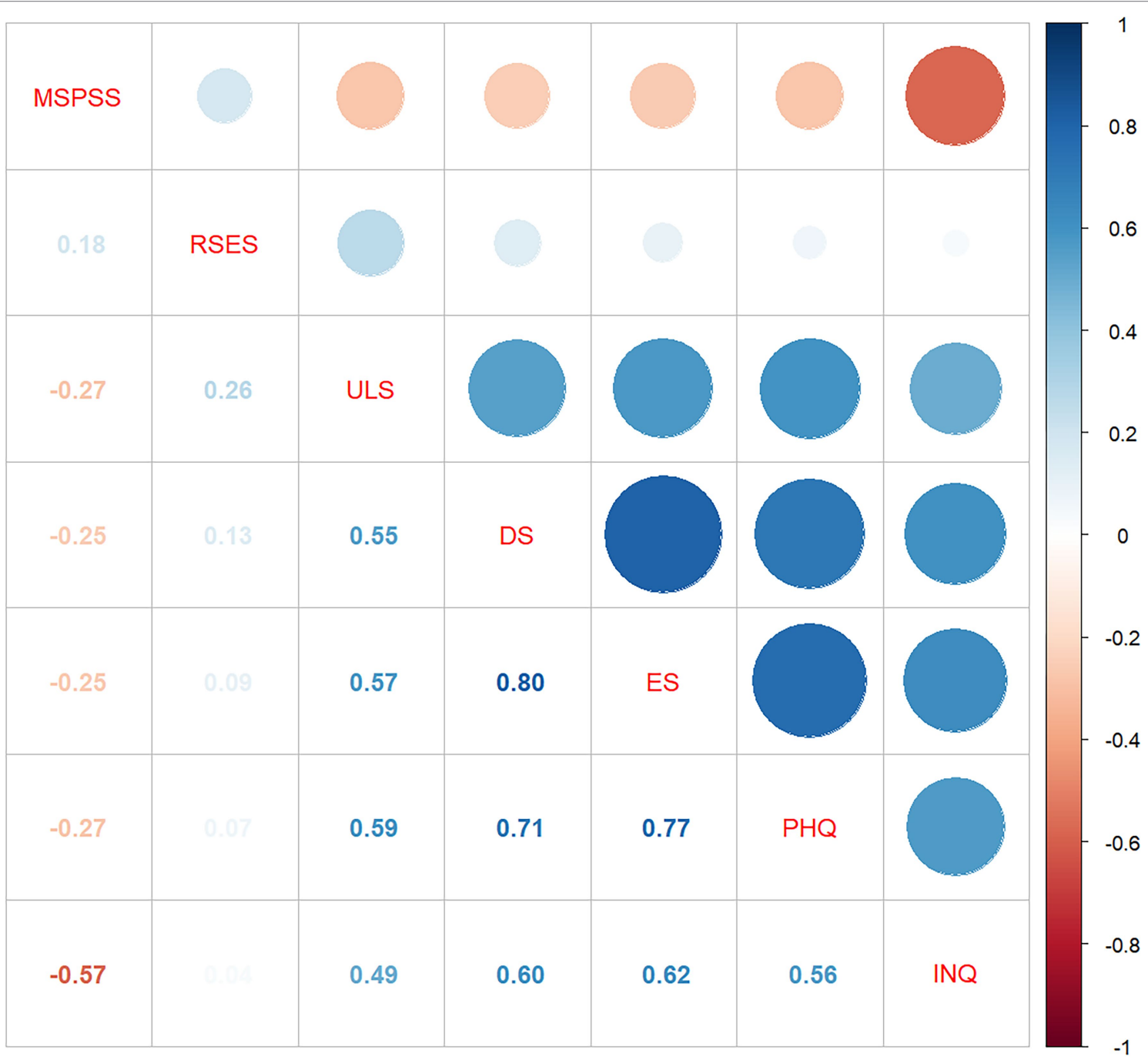

FIGURE 1 | Inter-correlations among psychosocial factors.

experiences (Van Orden et al., 2012). However, the present results showed that with the extraction of an additional factor, the different dimensions of the INQ could be clearly distinguished apart and social exclusion displayed stronger associations with those psychological measures than the other two dimensions. Although perceived burdensomeness was 


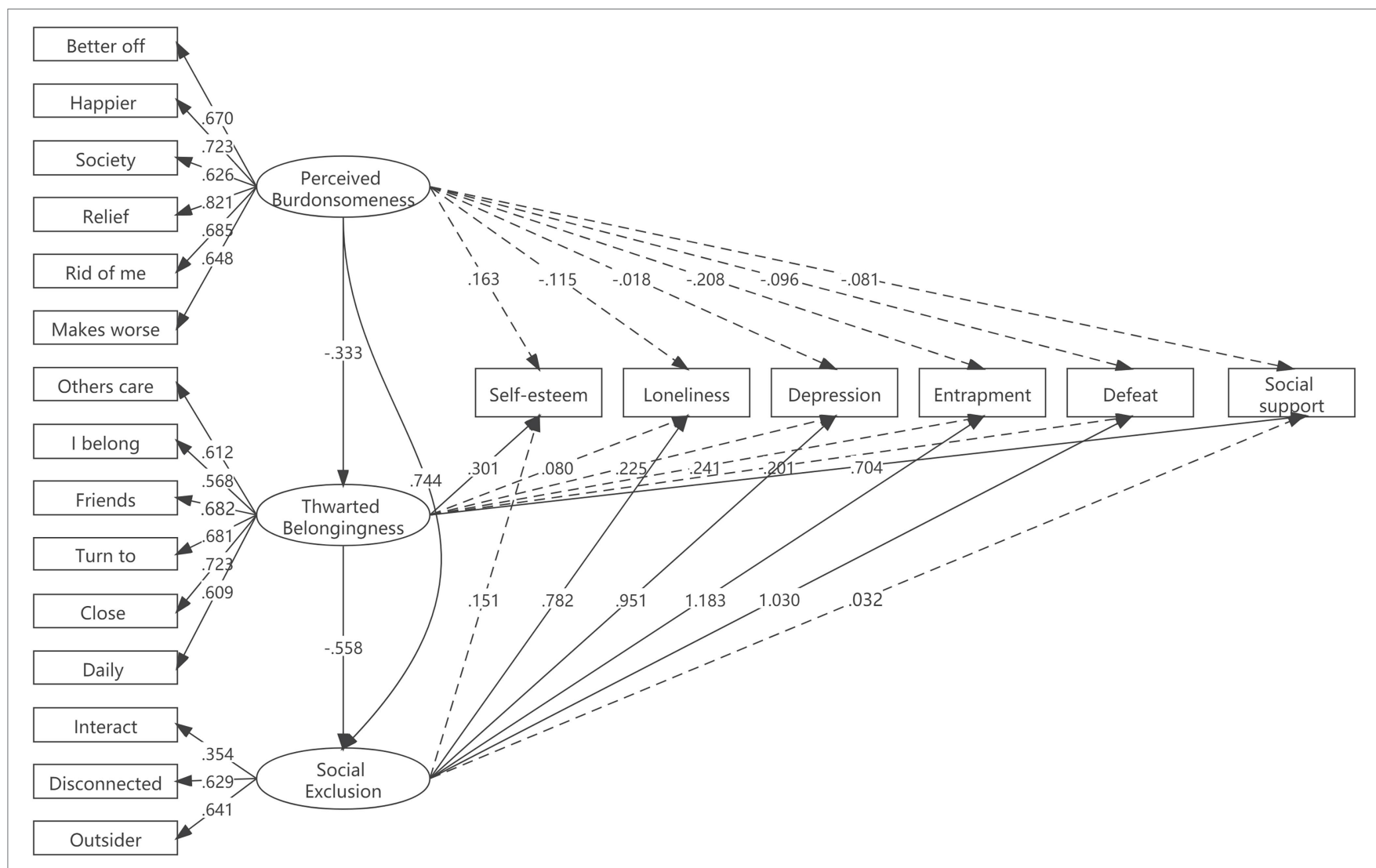

FIGURE 2 | Structural equation model results examining convergent and divergent validity.

correlated with none of these variables in the present study, this may not indicate poor psychometric properties; rather, perceived burdensomeness, which is characterized by the feeling of self-hatred and being like a burden to others, is related to other distinct factors including competence, autonomy, and responsibility to family (Van Orden et al., 2012). Further study is needed to identify constructs that are related to perceived burdensomeness and clarify the convergent validity for this factor.

There are at least 400,000 transgender people in China based on the reported gender-affirming surgery cases (Jiang et al., 2014). However, the socio-cultural environment is still hostile for transgender people in China, which expresses through a high level of discrimination and a lack of acceptance and protection (Peng et al., 2019). Transgender people bear a greater burden of poor mental health, among which TGW are at the highest risk of suicidal ideation and suicidal attempt (Chen et al., 2019). However, studies on this vulnerable population with regard to suicide risk are still limited in China (Chen et al., 2019). The present study validated among a sample of TGW in China the use of the INQ to measure perceived unmet interpersonal needs, which have been identified as the proximal causes of suicidal thoughts and behaviors and indicate the presence of suicidal ideation (Joiner, 2005; Van Orden et al., 2008). The INQ has the potential to be a valuable instrument for detecting suicide risks because individuals are more likely to accurately report their thoughts on the INQ in contrast to the direct suicide assessment, given their fear of involuntary hospitalization or feelings of shame (Van Orden et al., 2012). Therefore, future studies can utilize this scale to further explore the suicidal ideation and attempt in Chinese TGW, which may provide important evidence in the development of effective suicide screening programs and prevention interventions in this population.

There are several study limitations that should be acknowledged. First, the sample size was relatively small owing to the scarcity of TGW, thus we could not divide the final sample into two groups to conduct exploratory factor analysis and CFA separately. However, the present findings provide an insight into the mental health problems of this hard-to-reach population. Second, the cross-sectional nature of the present study limited the evaluation of the psychometric properties of the INQ. To further validate the use of the Chinese version of the INQ in TGW, additional longitudinal studies are needed to explore the test-retest reliability and predictive validity of this scale in TGW. Third, as the present sample was limited to TGW in Shenyang, we should be cautious when generalizing the results to countrywide conclusions. 


\section{CONCLUSION}

The INQ has good psychometric properties with three distinct but related dimensions of interpersonal needs among TGW: perceived burdensomeness, thwarted belongingness, and social exclusion. To our knowledge, this is the first study that validates the use of the Chinese version of the INQ in a sample of TGW, which may provide important implication for future study. First, TGW experience a high level of perceived social exclusion which is closely associated with unmet interpersonal needs, thus additional strategies should be developed to reduce discrimination and improve the perception of fitting in for TGW. Second, future researchers and clinicians can use the Chinese version of the INQ as a reliable and valid assessment tool to further study the perceived interpersonal needs and better assess the suicide risks among TGW.

\section{DATA AVAILABILITY STATEMENT}

The raw data supporting the conclusions of this article will be made available by the authors, without undue reservation.

\section{ETHICS STATEMENT}

The studies involving human participants were reviewed and approved by the Institutional Review Board of the School of Public Health, Shanghai Jiao Tong University School of Medicine. The patients/participants provided their written informed consent to participate in this study.

\section{REFERENCES}

Amini-Tehrani, M., Sadeghi, R., Nasiri, M., Jalali, T., and Zamanian, H. (2021). Suicide-related interpersonal needs of young Iranian people: a preliminary validation of thwarted belongingness and perceived burdensomeness constructs. Clin. Psychol. Psychother. 28, 422-437. doi: 10.1002/cpp.2507

Becasen, J. S., Denard, C. L., Mullins, M. M., Higa, D. H., and Sipe, T. A. (2019). Estimating the prevalence of HIV and sexual behaviors among the US transgender population: a systematic review and meta-analysis, 2006-2017. Am. J. Public Health 109, e1-e8. doi: 10.2105/AJPH.2018.304727

Becker, S. P., Foster, J. A., and Luebbe, A. M. (2020). A test of the interpersonal theory of suicide in college students. J. Affect. Disord. 260, 73-76. doi: 10.1016/j.jad.2019.09.005

Chen, R., Zhu, X., Wright, L., Drescher, J., Gao, Y., Wu, L., et al. (2019). Suicidal ideation and attempted suicide amongst Chinese transgender persons: national population study. J. Affect. Disord. 245, 1126-1134. doi: 10.1016/j.jad.2018.12.01

Chu, C., Buchman-Schmitt, J. M., Hom, M. A., Stanley, I. H., and Joiner, T. E. Jr. (2016). A test of the interpersonal theory of suicide in a large sample of current firefighters. Psychiatry Res. 240, 26-33. doi: 10.1016/j.psychres.2016.03.041

Gilbert, P., and Allan, S. (1998). The role of defeat and entrapment (arrested flight) in depression: an exploration of an evolutionary view. Psychol. Med. 28, 585-598. doi: 10.1017/S0033291798006710

Gong, R., Liu, J., Wang, Y., Cai, Y., and Wang, S. (2019). Validity and reliability of the Chinese vision of the Entrapment Scale in medical students. Chin. Ment. Health J. 33, 393-397. doi: 10.3969/j.issn.1000-6729.2019.05.015

\section{AUTHOR CONTRIBUTIONS}

YC, HW, RC, YW, and LJ: study design. HW, YC, and RC: data collection. CX, XY, SL, LJ, and HC: data curation. YL, RW, and YC: writing-original draft and formal analysis. YC, YW, LJ, and LX: writing-review and editing. All authors have read and agreed to the published version of the manuscript.

\section{FUNDING}

This work was supported by the National Natural Science Funds of China under Grant 71673187; the Shanghai Three-Year Action Plan for Public Health under Grants GWV-10.2-XD13, GWV-10.1-XK15, and GWV-10.1-XK18; the Strategic collaborative innovation team under Grant SSMU-ZLCX20180601; and the National Key Research and Development Project under Grants 2018YFC1705100 and 2018YFC1705103. The funding body had no role in the study design, collection, analysis, or interpretation of the data, writing the manuscript, or the decision to submit the paper for publication.

\section{ACKNOWLEDGMENTS}

We would like to thank all the participants who contributed to this research.

\section{SUPPLEMENTARY MATERIAL}

The Supplementary Material for this article can be found online at: https://www.frontiersin.org/articles/10.3389/fpsyg.2022.787809/ full\#supplementary-material

Hays, R. D., and DiMatteo, M. R. (1987). A short-form measure of loneliness J. Pers. Assess. 51, 69-81. doi: 10.1207/s15327752jpa5101_6

Hunt, Q. A., Weiler, L. M., McGuire, J., Mendenhall, T., Kobak, R., and Diamond, G. S. (2020). Testing basic assumptions of the interpersonal needs questionnaire-15 in a sample of clinically depressed and suicidal youth. Suicide Life Threat. Behav. 50, 372-386. doi: 10.1111/sltb.12594

Hyde, Z., Doherty, M., Tilley, M., McCaul, K., Rooney, R., and Jancey, J. (2013). The First Australian National Trans Mental Health Study: Summary of Results. Curtin University of Technology, School of Public Health.

Iliceto, P., D’Antuono, L., Fino, E., Carcione, A., Candilera, G., Silva, C., et al. (2021). Psychometric properties of the Italian version of the interpersonal needs questionnaire-15 (INQ-15-I). J. Clin. Psychol. 77, 268-285. doi: 10.1002/jclp.23026

Jiang, H., Wei, X., Zhu, X., Wang, H., and Li, Q. (2014). Transgender patients need better protection in China. Lancet 384, 2109-2110. doi: 10.1016/ s0140-6736(14)62372-2

Joiner, T. E. (2005). Why People Die by Suicide. Cambridge, MA: Harvard University Press.

Kroenke, K., Spitzer, R. L., and Williams, J. B. (2001). The PHQ-9: validity of a brief depression severity measure. J. Gen. Intern. Med. 16, 606-613. doi: 10.1046/j.1525-1497.2001.016009606.x

Lai, C. C. W., and Boag, S. (2021). Chinese versions of the interpersonal needs questionnaire: psychometric properties, measurement invariance across gender and cultures. PsyCh J. 10, 635-648. doi: 10.1002/pchj.436

Leung, D. Y. P., Mak, Y. W., Leung, S. F., Chiang, V. C. L., and Loke, A. Y. (2020). Measurement invariances of the PHQ-9 across gender and age groups in Chinese adolescents. Asia Pac. Psychiatry 12:e12381. doi: 10.1111/appy.12381 
Li, X., Xin, T., Yuan, J., Lv, L., Tao, J., and Liu, Y. (2015). Validity and reliability of the interpersonal needs questionnaire in Chinese college students. Chin. J. Clin. Psych. 23, 590-593. doi: 10.16128/j.cnki.1005-3611.2015.04.005

Liang, D., Xu, D., Xia, L., and Ma, X. (2020). Life satisfaction in Chinese rural-to-urban migrants: investigating the roles of self-esteem and affect balance. J. Community Psychol. 48, 1651-1659. doi: 10.1002/jcop.22360

Loas, G., Solibieda, A., Moens, K., Rotsaert, M., and Englert, Y. (2021). Interpersonal-psychological theory of suicide: study on 178 medical students. Ann. Med. Psycho. 179, 14-20. doi: 10.1016/j.amp.2020.04.014

McDonald, R. P., and Ho, M. H. (2002). Principles and practice in reporting structural equation analyses. Psychol. Methods 7, 64-82. doi: 10.1037/1082-989x.7.1.64

Muthén, L. K., and Muthén, B. O. (2002). How to use a Monte Carlo study to decide on sample size and determine power. Struct. Equ. Model. 9, 599-620. doi: 10.1207/S15328007SEM0904_8

Nemoto, T., Bödeker, B., Iwamoto, M., and Sakata, M. (2014). Practices of receptive and insertive anal sex among transgender women in relation to partner types, sociocultural factors, and background variables. AIDS Care 26, 434-440. doi: 10.1080/09540121.2013.841832

Nunnally, JC, and Bernstein, IH. (1994). Psychometric Theory. New York, NY: McGraw-Hill

O'Connor, S. S., Carney, E., Jennings, K. W., Johnson, L. L., Gutierrez, P. M., and Jobes, D. A. (2017). Relative impact of risk factors, thwarted belongingness, and perceived burdensomeness on suicidal ideation in veteran service members. J. Clin. Psychol. 73, 1360-1369. doi: 10.1002/jclp.22426

Park, Y., and Kim, H. S. (2019). Validation of the Korean version interpersonal needs questionnaire. Suicide Life Threat. Behav. 49, 739-758. doi: 10.1111/ sltb. 12473

Peng, K., Zhu, X., Gillespie, A., Wang, Y., Gao, Y., Xin, Y., et al. (2019). Selfreported rates of abuse, neglect, and bullying experienced by transgender and gender-nonbinary adolescents in China. JAMA Netw. Open 2:e1911058. doi: 10.1001/jamanetworkopen.2019.11058

Podlogar, T., Žiberna, J., Poštuvan, V., and C R Kerr D. (2017). Belongingness and burdensomeness in adolescents: Slovene translation and validation of the Interpersonal Needs Questionnaire. Suicide Life Threat. Behav. 47, 336-352. doi: $10.1111 /$ sltb. 12276

Poteat, T., Ackerman, B., Diouf, D., Ceesay, N., Mothopeng, T., Odette, K-Z., et al. (2017). HIV prevalence and behavioral and psychosocial factors among transgender women and cisgender men who have sex with men in 8 African countries: a cross-sectional analysis. PLoS Med. 14:e1002422. doi: 10.1371/ journal.pmed.1002422.

Poteat, T., Wirtz, A. L., Radix, A., Borquez, A., Silva-Santisteban, A., Deutsch, M. B., et al. (2015). HIV risk and preventive interventions in transgender women sex workers. Lancet 385, 274-286. doi: 10.1016/S0140-6736(14)60833-3

Rosenberg, M. (1989). Society and the Adolescent Self-Image (Rev. Edn.). Middeltown, CT: Wesleyan University Press.

Rust, J., and Golombok, S. (1999). Modern Psychometrics. London, New York: Routledge.

Shi, X., and Cai, Y. (2020). Progress of research on risk of HIV infection and related factors among transgender women. Chin. J. AIDS STD 26, 106-109+96. doi: 10.13419/j.cnki.aids.2020.01.29

Shim, Y., Choe, K., Kim, K. S., Kim, J. S., and Ha, J. (2021). The applicability of the interpersonal-psychological theory of suicide among community-dwelling older persons. Suicide Life Threat. Behav. 51, 816-823. doi: 10.1111/sltb.12757

Silva, C., Hurtado, G., Hartley, C., Rangel, J. N., Hovey, J. D., Pettit, J. W., et al. (2018). Spanish translation and validation of the interpersonal needs questionnaire. Psychol. Assess. 30, e21-e37. doi: 10.1037/pas0000643

Tang, H., Wang, S., Gong, R., Wang, Z., and Cai, Y. (2019). Reliability and validity of defeat scale on anxiety and depression in medical students. J. Shanghai Jiao Tong Univ. 39, 84-88. doi: CNKI:SUN:SHEY.0.2019-01-019

Teo, D. C. H., Suárez, L., and Oei, T. P. S. (2018). Validation of the interpersonal needs questionnaire of young male adults in Singapore. PLoS One 13:e0198839. doi: 10.1371 /journal.pone.0198839
Tsai, F. J., Huang, Y. H., Liu, H. C., Huang, K. Y., Huang, Y. H., and Liu, S. I. (2014). Patient health questionnaire for school-based depression screening among Chinese adolescents. Pediatrics 133, e402-e409. doi: 10.1542/ peds.2013-0204

Van Orden, K. A., Cukrowicz, K. C., Witte, T. K., and Joiner, T. E. (2012). Thwarted belongingness and perceived burdensomeness: construct validity and psychometric properties of the interpersonal needs questionnaire. Psychol. Assess. 24, 197-215. doi: 10.1037/a0025358

Van Orden, K. A., Witte, T. K., Cukrowicz, K. C., Braithwaite, S. R., Selby, E. A., and Joiner, T. E. Jr. (2010). The interpersonal theory of suicide. Psychol. Rev. 117, 575-600. doi: 10.1037/a0018697

Van Orden, K. A., Witte, T. K., Gordon, K. H., Bender, T. W., and Joiner, T. E. Jr. (2008). Suicidal desire and the capability for suicide: tests of the interpersonal-psychological theory of suicidal behavior among adults. J. Consult. Clin. Psychol. 76, 72-83. doi: 10.1037/0022-006X.76.1.72

Wang, M., Xu, Q., and He, N. (2021b). Perceived interparental conflict and problematic social media use among Chinese adolescents: the mediating roles of self-esteem and maladaptive cognition toward social network sites. Addict. Behav. 112:106601. doi: 10.1016/j.addbeh.2020.106601

Wang, R., Chen, Y., Hu, F., Wang, Z., Cao, B., Xu, C., et al. (2021a). Psychometric properties of interpersonal needs questionnaire-15 for predicting suicidal ideation among migrant industrial workers in China. Int. J. Environ. Res. Public Health 18:7583. doi: 10.3390/ijerph18147583

Winter, S., Diamond, M., Green, J., Karasic, D., Reed, T., Whittle, S., et al. (2016). Transgender people: health at the margins of society. Lancet 388, 390-400. doi: 10.1016/S0140-6736(16)00683-8

White Hughto, J. M., Reisner, S. L., and Pachankis, J. E. (2015). Transgender stigma and health: a critical review of stigma determinants, mechanisms, and interventions. Soc. Sci. Med. 147, 222-231. doi: 10.1016/j. socscimed.2015.11.010

Xu, S., Qiu, D., Hahne, J., Zhao, M., and Hu, M. (2018). Psychometric properties of the short-form UCLA loneliness scale (ULS-8) among Chinese adolescents. Medicine 97:e12373. doi: 10.1097/MD.0000000000012373

Zhang, M., Zhang, J., Zhang, F., Zhang, L., and Feng, D. (2018). Prevalence of psychological distress and the effects of resilience and perceived social support among Chinese college students: does gender make a difference? Psychiatry Res. 267, 409-413. doi: 10.1016/j.psychres.2018.06.038

Zhou, L., Li, Z., Hu, M., and Xiao, S. (2012). Reliability and validity of ULS-8 loneliness scale in elderly samples in a rural community. Zhong Nan Da Xue Xue Bao Yi Xue Ban 37, 1124-1128. doi: 10.3969/j.issn.1672-7347. 2012.11.008

Zimet, G. D., Dahlem, N. W., and Zimet, S. G. (1988). The multidimensional scale of perceived social support. J. Pers. Assess. 52, 30-41. doi: 10.1207/ s15327752jpa5201_2

Conflict of Interest: The authors declare that the research was conducted in the absence of any commercial or financial relationships that could be construed as a potential conflict of interest.

Publisher's Note: All claims expressed in this article are solely those of the authors and do not necessarily represent those of their affiliated organizations, or those of the publisher, the editors and the reviewers. Any product that may be evaluated in this article, or claim that may be made by its manufacturer, is not guaranteed or endorsed by the publisher.

Copyright (c) 2022 Liu, Wang, Chang, Wang, Xu, Xu, Yu, Liu, Chen, Chen, Jin, Wang and Cai. This is an open-access article distributed under the terms of the Creative Commons Attribution License (CC BY). The use, distribution or reproduction in other forums is permitted, provided the original author(s) and the copyright owner(s) are credited and that the original publication in this journal is cited, in accordance with accepted academic practice. No use, distribution or reproduction is permitted which does not comply with these terms. 